\title{
Performance comparison between overlapped and woven spacers for membrane distillation
}

\author{
Luigi Gurreri, Alessandro Tamburini*, Andrea Cipollina, Giorgio Micale, Michele Ciofalo \\ Dipartimento dell'Innovazione Industriale e Digitale (DIID) - Ingegneria Chimica, Gestionale, Informatica, Meccanica, Università \\ degli Studi di Palermo (UNIPA) - viale delle Scienze Ed.6, 90128 Palermo, Italy, email: luigi.gurreri@unipa.it (L. Gurreri), \\ alessandro.tamburini@unipa.it (A. Tamburini),andrea.cipollina@unipa.it (A.Cipollina), giorgiod.maria.micale@unipa.it (G. Micale), \\ michele.ciofalo@unipa.it (M. Ciofalo)
}

Received 26 July 2016; Accepted 20 September 2016

\section{A B S T R A C T}

The sustainable production of freshwater from seawater desalination is receiving increasing attention. Recently, some desalination technologies are taking advantage from the coupling with renewable resources; among them, membrane distillation (MD) is one of the most promising since it can be easily powered by low-grade thermal energy. MD being an emerging technology, efforts are required to optimize geometry and operating conditions of real units in order to reduce the unitary freshwater production cost. In particular, temperature polarization is a well-known detrimental effect for the process driving force; spacers are traditionally used to enhance mixing and make temperature boundary layers thinner, at the cost of higher pressure losses. The present work is devoted to testing and comparing the performance of two different two-layer net spacers: overlapped and woven Investigations were carried out both by experiments and by computational fluid dynamics (CFD) at different Reynolds numbers, ranging from creeping flow to turbulent flow regimes. Experiments (for intermediate to high Re) made use of thermochromic liquid crystals along with digital image processing. Computational results (for low to intermediate Re) were obtained via steady state (low Re) or direct numerical simulations (intermediate Re) along with the unit cell approach. A good agreement between experiments and CFD results was obtained in the range of superposition. Results showed that woven spacers guarantee a better mixing than overlapped ones, especially in the low to intermediate Re range, thus resulting in Nusselt numbers 2.5-3 times higher. On the other hand, the less disturbed flow field induced by overlapped spacers was found to yield friction coefficients up to 4 times lower, thus allowing lower pumping costs. The choice between the two configurations depends crucially on the relative importance attributed to savings in membrane surface area and in pumping energy for any specific application.

Keywords: Membrane distillation; Computational fluid dynamics; Spacer-filled channels; Thermochromic liquid crystals

\section{Introduction}

Membrane distillation (MD) has attracted much attention in the last decades [1]. Its main attractive feature is that the process can be driven by low grade thermal energy [2],

${ }^{*}$ Corresponding author. which makes it a potentially attractive candidate for pairing with renewable energy sources, such as solar thermal or geothermal energy, and/or taking advantage of waste heat, if any of these are available [3-10].

In $\mathrm{MD}$, a negative vapour pressure gradient is simply maintained between two sides of a microporous hydrophobic membrane, causing a net flux of vapour from the warm

Presented at the EDS conference on Desalination for the Environment: Clean Water and Energy, Rome, Italy, 22-26 May 2016. 
saline feed side to the cooler permeate side [11]. The vapour transported through the hydrophobic membrane is then condensed, either directly onto a cooler stream on the permeate side, or on a separate internal or external condensation surface. The specific energy required by the process does not quickly rise with the salinity of the feed as it occurs, for example, in the case of reverse osmosis [12]; this allows MD to be applied to desalinate very high salinity feed waters, and opens up opportunities for hybrid technology applications.

Not all of the applied temperature difference is utilised as a driving force. The reason is that, when fluids flow on both sides of the membrane, the trans-membrane temperature difference is lower than the difference between the bulk temperatures of feed and permeate (Fig. 1).

This temperature polarization effect is the result of convective heat transfer resistances on both sides and it is usually quantified by the temperature polarization coefficient, defined as the ratio between the trans-membrane temperature difference and the difference between the bulk temperatures of the two streams:

$$
T P C=\frac{T_{f m}-T_{p m}}{T_{f b}-T_{p b}}
$$

in which $f$ and $p$ refer to feed and permeate while $m$ and $b$ refer to membrane surface and fluid bulk, respectively. This coefficient increases with the Reynolds number, especially for low $\mathrm{Re}$, and decreases with the feed temperature [13].

Salt concentration polarization is also present in MD feed channels, but it has been shown that it plays only a minor role with respect to temperature polarization [14,15].

Much research effort is currently directed towards improved membranes, tailored to yield increasingly higher vapour fluxes [16]. However, as the membrane performance increases, the issue of temperature polarization acquires an increasing importance since it may become the limiting factor for performance, and thus the issue of module hydro-

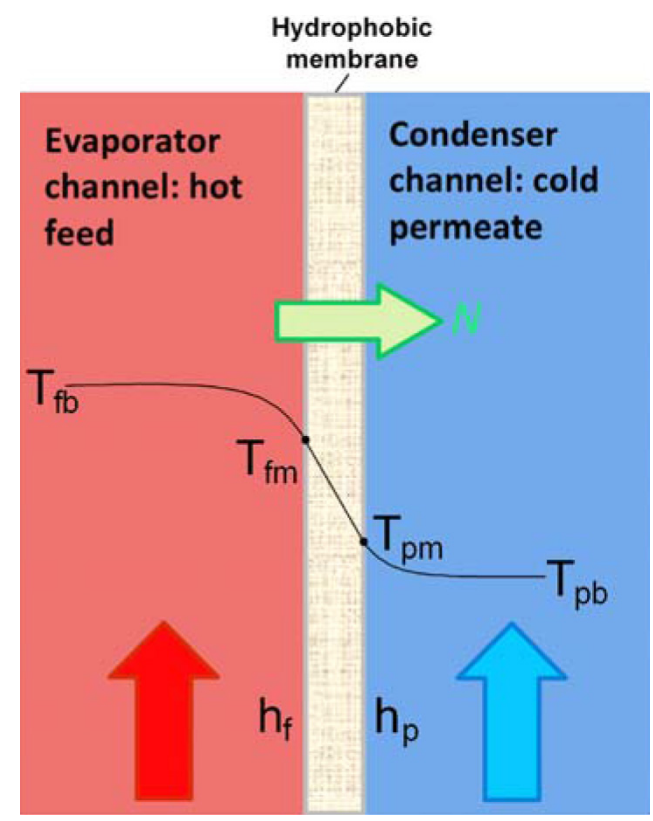

Fig. 1. Sketch of the temperature polarization effect in membrane distillation. dynamics has to be more and more carefully addressed. The principal means by which polarization is controlled in flat membrane configurations (including plate \& frame and spiral wound modules) are channel spacers. These typically consist of a net of polymeric filaments of varying geometrical features, and play an important role both in promoting mixing and in guaranteeing the dimensional stability of the channel. Unfortunately, spacers cause also larger hydraulic losses $[17,18]$, so that a compromise must be sought.

\section{Description of the problem and configurations investigated}

The spacer geometries investigated in the present paper are illustrated in Fig. 2. Fig. 2(a) refers to overlapped spacers, Fig. 2(b) to woven spacers.

In both cases, the pitch to channel height ratio was 2 . The spacer filaments (or, in the woven case, their projections on the horizontal plane) were mutually orthogonal. The porosity (fluid volume/total volume) of the spacer-filled channel was 0.82 for the overlapped configuration and 0.75 for the woven one. Heat transfer occurred only from the top wall, the opposite one being (to all practical purposes) adiabatic.

In all spacers, the flow attack angle $\theta$ is responsible for changes in flow direction and flow pattern which affect polarization and heat transfer [19] and thus deserves a particular attention. It was defined here as the angle formed by the main flow direction with one of the filament arrays (woven spacers) or with the filament array adjacent to the top, thermally active, wall (overlapped spacers). In these latter spacers, three values of $\theta$ were investigated $\left(0^{\circ}, 45^{\circ}\right.$ and $90^{\circ}$ ); note that the two angles of $0^{\circ}$ and $90^{\circ}$ were equivalent from the point of view of fluid dynamics (and, in fact, yielded the same friction coefficient), but not from the thermal point of view due to the inherent top-down asymmetry of the thermal boundary conditions. In woven spacers, only two values of $\theta\left(0^{\circ}\right.$ and $\left.45^{\circ}\right)$ were investigated because the top-wall distribution of any thermal quantity (tempera-

(a)

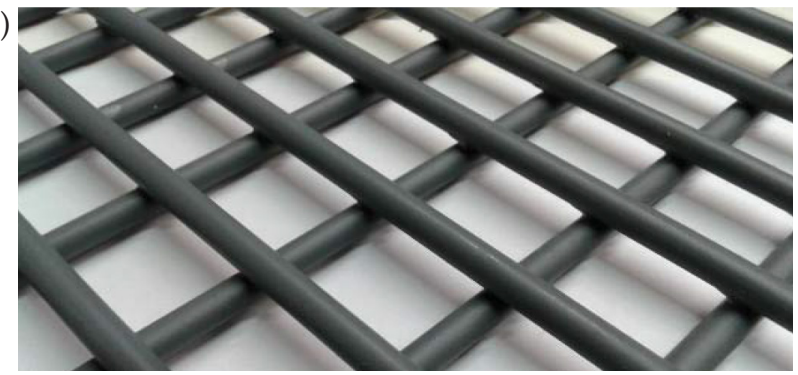

(b)

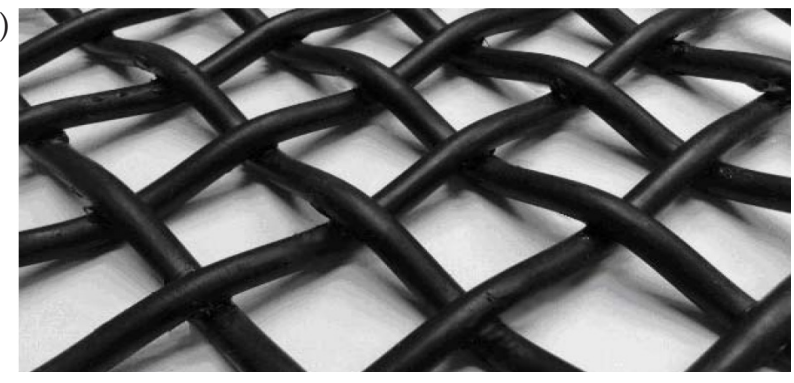

Fig. 2. Overlapped (a) and woven (b) spacers. 
ture, heat flux, heat transfer coefficient) remains unchanged under a $90^{\circ}$ rotation of the flow direction (apart from rotations and reflections); in particular, wall averaged heat transfer coefficients are the same for $\theta=0^{\circ}$ or $90^{\circ}$.

In the experiments, the flow attack angle was made to vary by building an appropriately oriented model spacer for each required value of $\theta$.

In the present study, dimensionless quantities are defined with reference to the corresponding void (spacerless) configuration, i.e. to a plane channel having height $H$ and thus hydraulic diameter $D_{h}=2 H$. Coherently, the bulk Reynolds number is defined as

$\operatorname{Re}=\mathrm{U} \cdot 2 \frac{H}{v}$

in which $U=Q /(W H)$ is the mean "void channel" streamwise velocity, being $Q$ the volume flow rate and $W$ the channel's spanwise extent (in the experiments, $W \approx 0.24 \mathrm{~m}$ ), and $v$ is the cinematic viscosity. In our experience, this scaling is preferable to that based on the actual hydraulic diameter and mean velocity of each specific case, and makes comparisons between different configurations easier and more meaningful. The friction velocity Reynolds number $\operatorname{Re}_{\tau}$ is defined as

$$
\operatorname{Re}_{\tau}=u_{\tau} \frac{\left(\frac{H}{2}\right)}{v}
$$

in which $u_{\tau}=\left(\left\langle\tau_{w}\right\rangle / \mathrm{r}\right)^{1 / 2}$ is the friction velocity. The quantity $\left\langle\tau_{w}\right\rangle$ is the wall-averaged wall shear stress which, in a void channel, would balance the large-scale pressure gradient along the main flow direction $\xi$, defined as $p_{\xi}=\Delta p / \Delta \xi$; i.e., one has $\left\langle\tau_{w}\right\rangle=(H / 2) p_{\varepsilon}$.

The Darcy friction coefficient (four times the Fanning friction factor) is defined as

$$
f=\frac{4 H}{\rho U^{2}} p_{\xi}
$$

On the basis of the definitions given above for Re and $\operatorname{Re}_{\tau^{\prime}}$ one has $f=128\left(\operatorname{Re}_{\tau} / \operatorname{Re}\right)^{2}$.

The local Nusselt number is defined as

$$
N u=h \frac{2 H}{k}
$$

in which $h=q^{\prime \prime}{ }_{w} /\left(T_{b}-T_{w}\right)$ is the local heat transfer coefficient, $q^{\prime \prime}{ }_{w}$ being the wall heat flux, $T_{b}$ the fluid bulk temperature and $T_{w}$ the local wall temperature, and $k$ is the thermal conductivity. Surface averages on the walls will be indicated with $\langle h\rangle$ and $\langle N u\rangle$.

\section{Literature review}

Starting with the work of Schock and Miquel [18], Da Costa et al. [17], Li et al. [20], much research effort has been devoted to understanding the effects of spacer geometry on fluid dynamics and heat or mass transfer in membrane-based processes involving cross flow in plane or spiral-wound modules.

In particular, for the case of MD, Velázquez and Mengual [21] carried out experiments to evaluate the temperature polarization coefficient in spacer-filled channels. Martinez-Diez et al. [22] and Yun et al. [23] stated that coarser spacers with thicker filaments perform better in reducing polarization and enhancing mass flux. Phattaranawik et al. [24] found flux enhancements up to $41 \%$ in spacer-filled channels compared to the empty channel. In a later paper [25] the same authors tested a number of existing correlations for laminar and turbulent flow conditions finding that: (i) the effects of mass transfer on heat transfer rates can be neglected, and (ii) the significance of heat conduction in the membranes and of heat transfer due to the vapour flow was comparable. Following such findings, the authors [26] refused the adoption of the analogy between heat and mass transfer and proposed a new correlation more suitable to the case of MD. Chernyshov et al. [27] investigated experimentally the effect of spacer geometry on process performance for the case of air gap membrane distillation: when a spacer was employed within the channel, a mass flux up to 2.5 times higher than in the spacerless channel was found. However, due to the relevant pressure drop increase, the spacerless channel was found to provide the highest flux at a given pressure drop. A model to characterize a direct contact membrane distillation module was developed by Martínez-Diez and Rodríguez-Maroto [28], aiming at determining the mass and heat transfer coefficients through the membrane and the boundary layers. Significant heat transfer improvements were found by the authors in the spacer-filled channel $(>50 \%)$ both by the model and by experiments.

Currently, the selection of channel spacers in the context of membrane distillation is often based on commercially available models, rather than on customised designs supported by detailed thermofluid dynamics considerations. Given the important role of channel spacers, there is a definite need for rational methodologies for spacer selection and/or development. Ideally, the desired methodology would provide local spatial resolution, revealing the detailed hydrodynamic and heat/mass transfer features of a candidate spacer. This aspect is available in computational fluid dynamics (CFD), which, in principle, can provide complete detail of all features of the fluid flow and heat transfer. Indeed there have been several studies applying CFD to spacer-filled channels for MD [29-32], and these have generally demonstrated the great deal of useful and relevant information that can be extracted from CFD simulations. What is lacking at this point is thorough experimental validation. This is mainly because available experimental techniques are usually able to provide measurements of spatially averaged quantities only or, at best, of $1 \mathrm{D}$ variations.

One example of measurement of local quantities is the work of Ali et al. [13]. They analysed heat and mass transfer in direct contact membrane distillation (MD) by using a cell equipped with sixteen sensors in order to measure the bulk and membrane surface temperatures. A more comprehensive characterization of spacers' behaviour would require a technique able to obtain the distribution of temperature with a higher spatial resolution.

The technique employed in the present work addresses the requirement of a high spatial resolution of the temperature field through the use of liquid crystal thermography along with digital image analysis. It has been presented 
in detail in previous papers $[33,34]$; therefore, only a brief account will be given in the following Section.

\section{Experimental technique}

In a real membrane distillation unit, heat transfer from the hot fluid (feed) to the cold fluid (permeate) is a threestep process: (a) transfer of sensible heat from the bulk feed to the feed-membrane interface by conduction and convection; (b) transfer of sensible and latent heat through the membrane, the latter component being associated with mass transfer (vapour flux); and, (c) heat transfer, mainly by condensation, to the permeate (this latter step depending on the exact configuration chosen, i.e. direct condensation, permeate gap, air gap etc.). The focus of the present study is on the first of the above steps, in which polarization issues occur and the use of mixing promoters may be effective. Since this step involves only the conductive and convective transfer of sensible heat through the feed, it can be experimentally investigated with accuracy even if the other steps (involving a permeable membrane and a condensate channel) are not present: all that is required is a heat flux from the feed to a solid wall, sustained by a temperature gradient. The magnitude of this flux, and thus the thermal resistances of wall and permeate, have little or no influence on the feed side heat transfer coefficient.

On the basis of the above rationale, in the present experiments the feed was simulated by hot water, the membrane by a thermally conductive and optically transparent (as required by the measurement technique) polycarbonate wall, and the permeate by cold water. Of course, no mass transfer occurred.

The heart of the present measurement technique is liquid crystal thermography. Thermochromic liquid crystals (TLC) are organic compounds whose colour varies with temperature. Commercially available TLCs can come in various forms, such as spray paints and flat strips. In the present technique, flat strips of TLC sheets are used to measure the local surface temperature distribution in a specific controlled region.

The experimental test section consists of two flat flow passages delimited by two thick $(20 \mathrm{~mm})$ transparent Plexiglas ${ }^{\circledast}$ plates, which provide structural stiffness and thermal insulation, and by a thin $(1 \mathrm{~mm})$ transparent polycarbonate sheet. Hot water flows in the bottom channel, where the spacer to be tested is placed. Cold water flows in the top channel. The height of the two flow passages is determined by either the spacer to be tested (hot channel) or by the appropriately sized gaskets (cold channel). As anticipated in Section 2, the flow attack angle was made to vary by building an appropriately oriented model spacer for each required value of $\theta$.

TLC strips (manufactured by Hallcrest ${ }^{\circledR}$ ) are affixed to the polycarbonate sheet on its hot channel side, in contact with the spacer, by interposing a thin layer of non-adhesive silicone grease. No spacer is used in the cold channel.

Fig. 3 shows a schematic layout of the test section (a) and a photograph in the working configuration (b).

Two pressure tappings, located along the midline of the bottom channel and $50 \mathrm{~cm}$ apart, are connected to a differential pressure transducer (Fuji Electric FCX-AII ${ }^{\circledR}$ Series) to
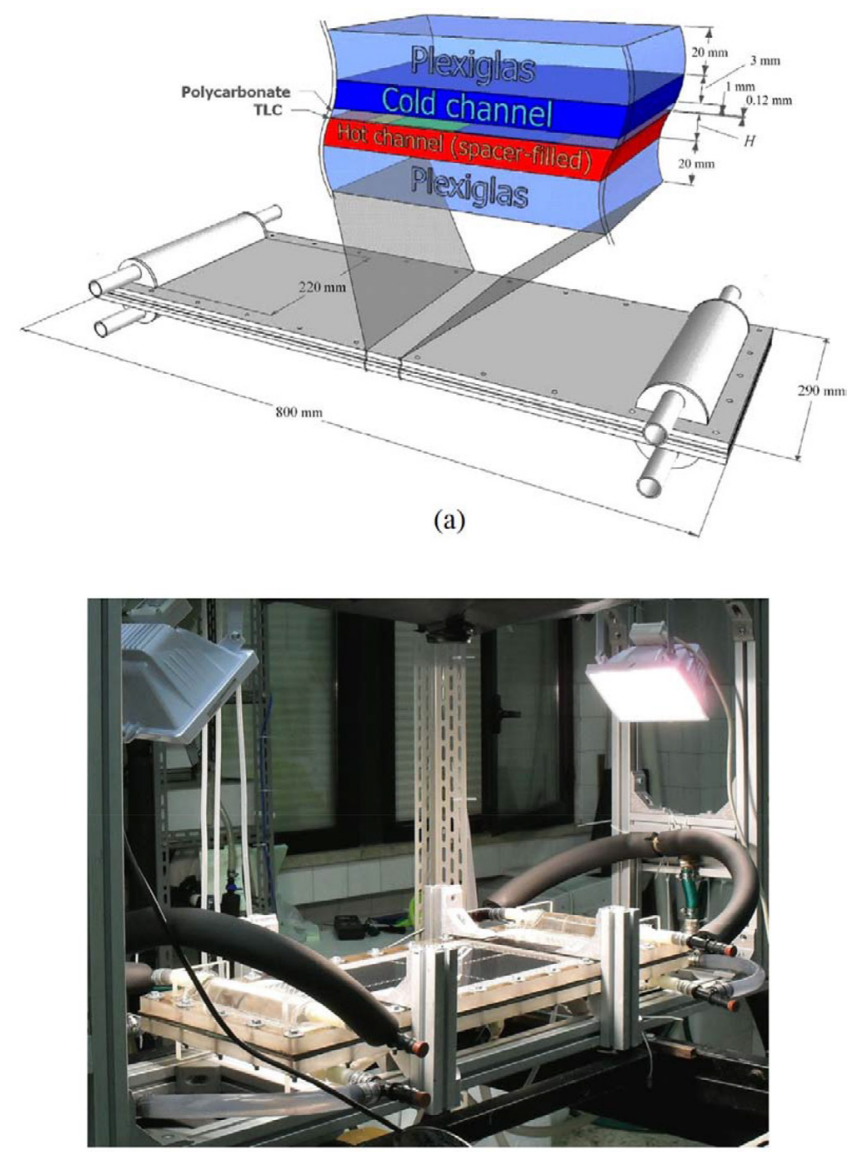

(b)

Fig. 3. Test section: (a) schematic view, showing a magnified layout of the cross section; (b) photograph in the working configuration, showing light sources, camera and TLC strips.

allow pressure drops to be measured. The Plexiglas ${ }^{\circledR}$ test section is fixed in place on an aluminium frame structure, which also supports a digital camera (Canon EOS $550 \mathrm{D}^{\circledR}$ ) and two light sources.

A chiller (Corema ${ }^{\circledR}$ Junior Chiller JA/C 150) and a thermostatic heating bath (Julabo ${ }^{\circledR}$ ED-5) are used to control the temperatures of the cold and warm flows, respectively. The desired flow rates are supplied by two recirculation pumps with flow control bypass valves, and the two flow rates are measured using two magnetic flow meters (Krohne ${ }^{\circledR}$ Optiflux 5300 C). The inlet and outlet temperatures of both flows are measured using four Pt100 probes, which transmit their readings to a National Instruments ${ }^{\circledR}$ data acquisition system.

TLC strips need to be calibrated to determine the exact colour to temperature relationship. Although digital cameras usually output images in the RGB (red, green, blue) colour space, it is more convenient to transform the images into the HSL (Hue-Saturation-Luminance) colour space representation, because only hue correlates with temperature. An in-situ calibration was performed according to the steady-state method of uniform surface temperature [35]: water supplied by the heating circulator was forced to flow through the channels on both sides 
of the TLC-polycarbonate wall, and its temperature was progressively increased from $30^{\circ} \mathrm{C}$ up to $42^{\circ} \mathrm{C}$ in $0.2^{\circ} \mathrm{C}$ steps. For each imposed temperature, once steady state conditions were achieved in the module, a photograph of the colorimetric response of the TLCs was acquired, thus resulting into a hue vs temperature $(\mathrm{H}-\mathrm{T})$ calibration curve. Piecewise polynomials were employed to fit the data. Of course, all the following tests were carried out at temperatures falling within the range of calibration $\left(35-40^{\circ} \mathrm{C}\right)$.

Fig. 4 reports two typical test images, respectively for an overlapped spacer at a flow attack angle $\theta$ of $90^{\circ}$ (a) and for a woven spacer at $\theta=45^{\circ}(\mathrm{b})$.

All the test images were processed by the Matlab $^{\circledR}$ Image Processing Toolbox. TLC pictures like those in Fig. 4, acquired by the camera in the RAW format for maximum quality, were first converted into the TIFF format and suitably cropped. They were then converted from RGB to HSL. Finally, the calibration curve allows Hue data to be converted into temperature data.

If the bulk temperatures of both fluids and the cold side heat transfer coefficient $h$ are known, the hot-side heat transfer coefficient $h_{h}$ can be inferred from the temperature $T_{w}$ of the TLC sheet. With reference to the temperature profiles outlined in Fig. 5 (which also reports the relevant nomenclature), by assuming one-dimensional heat transfer, $h_{h}$ can be computed as follows:

$$
h_{h}=\frac{T_{w}-T_{c}}{\left(T_{h}-T_{w}\right)\left(\frac{L_{T L C}}{k_{\text {TLC }}}+\frac{L_{\text {poly }}}{k_{\text {poly }}}+\frac{1}{h_{c}}\right)}
$$

in which $L$ is thickness and $k$ is thermal conductivity. The soundness of the one-dimensional transfer hypothesis was already demonstrated in a previous work [33]. Although Eq. (6) contains the (unknown) cold-side heat transfer coefficient $h_{c^{\prime}}$ it was verified that, provided $h_{c} \gg h_{h^{\prime}}$ the computed value of $h_{h}$ is little sensitive to the value assumed for $h_{c}$. Therefore, $h_{c}$ was simply computed by the Dittus-Bölter heat transfer correlation on the basis of the cold channel thickness and cold fluid flow rate.

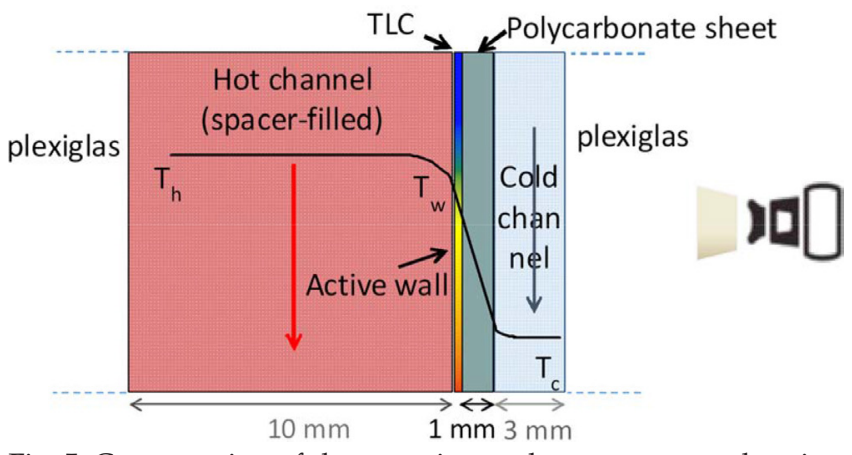

Fig. 5. Cross section of the experimental arrangement, showing the position of the camera and the temperatures used for the assessment of the hot-side heat transfer coefficient $h_{h}$.

\section{Computational method}

In the present study, the computational domains were the unit cells shown in Fig. 6(a) for the overlapped spacer and in Fig. 6(b) for the woven one. Note that the latter unit cell is twice larger than the former, so that periodicity conditions could be imposed at its boundaries.

Mathematically, the problem was described by the continuity, Navier-Stokes and energy equations for a constant-property fluid. The "unit cell" treatment, discussed in previous papers [36], allowed periodicity conditions to be adopted for all variables at the opposite faces of the computational domain. All simulations were conducted by the finite volume ANSYS-CFX14 ${ }^{\circledR}$ code [37].

No slip conditions were imposed at the top and bottom walls and on the filaments' surface. As regards the thermal boundary conditions, the bottom wall and the filaments were assumed to be adiabatic $\left(q_{w}^{\prime \prime}=0\right)$, while at the top wall a Cauchy (mixed) condition was imposed:

$T_{\mathrm{w}}-T_{\mathrm{c}}=r q^{\prime \prime}{ }_{\mathrm{w}}$

mimicking the experimental boundary condition, with outer temperature $T_{c}\left(19^{\circ} \mathrm{C}\right)$ and thermal resistance $r\left(6.25 \cdot 10^{-3} \mathrm{~m}^{2}\right.$ $\mathrm{K} / \mathrm{W})$ representative of the experiments. Also the spacer sizes were the same as the experimental ones. The fluid was assumed to be water at $39^{\circ} \mathrm{C}$ with physical properties $r=$

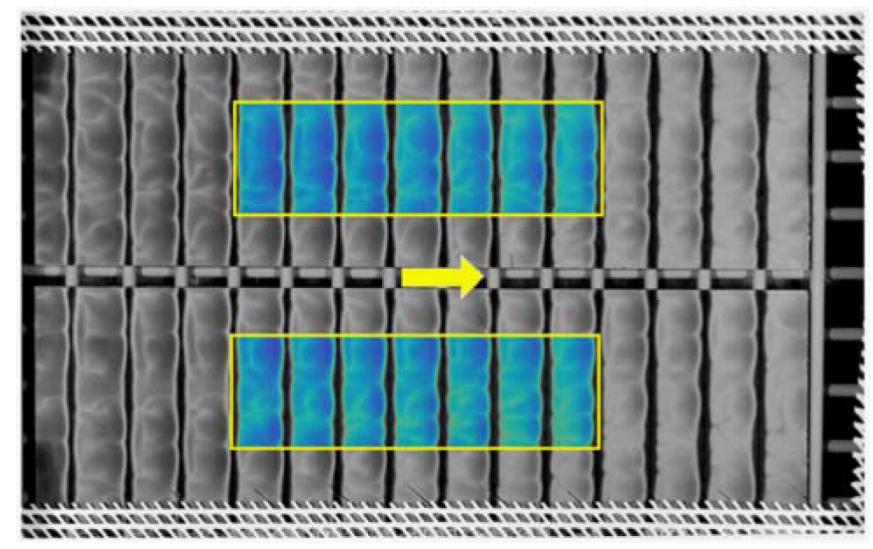

(a)

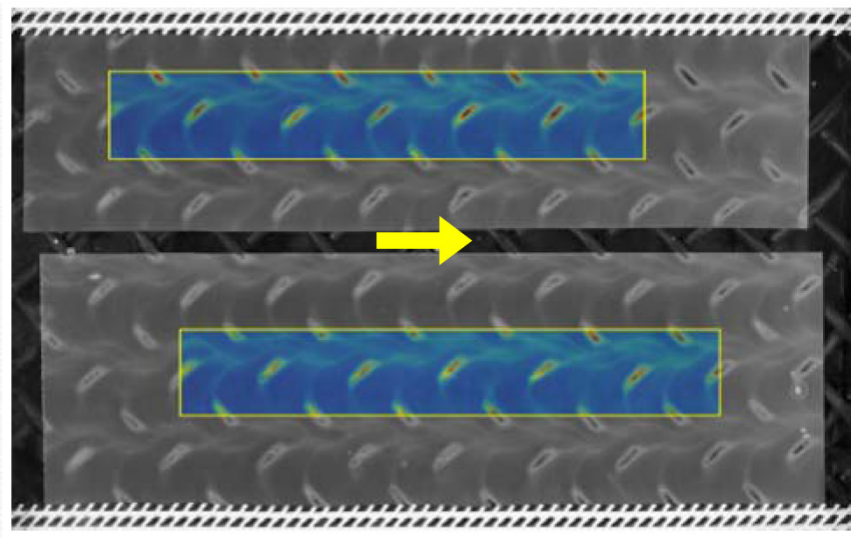

(b)

Fig. 4. Typical TLC images: (a) overlapped spacer at a flow attack angle $\theta=90^{\circ}$; (b) woven spacer at a flow attack angle $\theta=45^{\circ}$. Flow is from left to right (yellow arrows). The insets in color indicate the portions of the TLC images that were actually processed. 

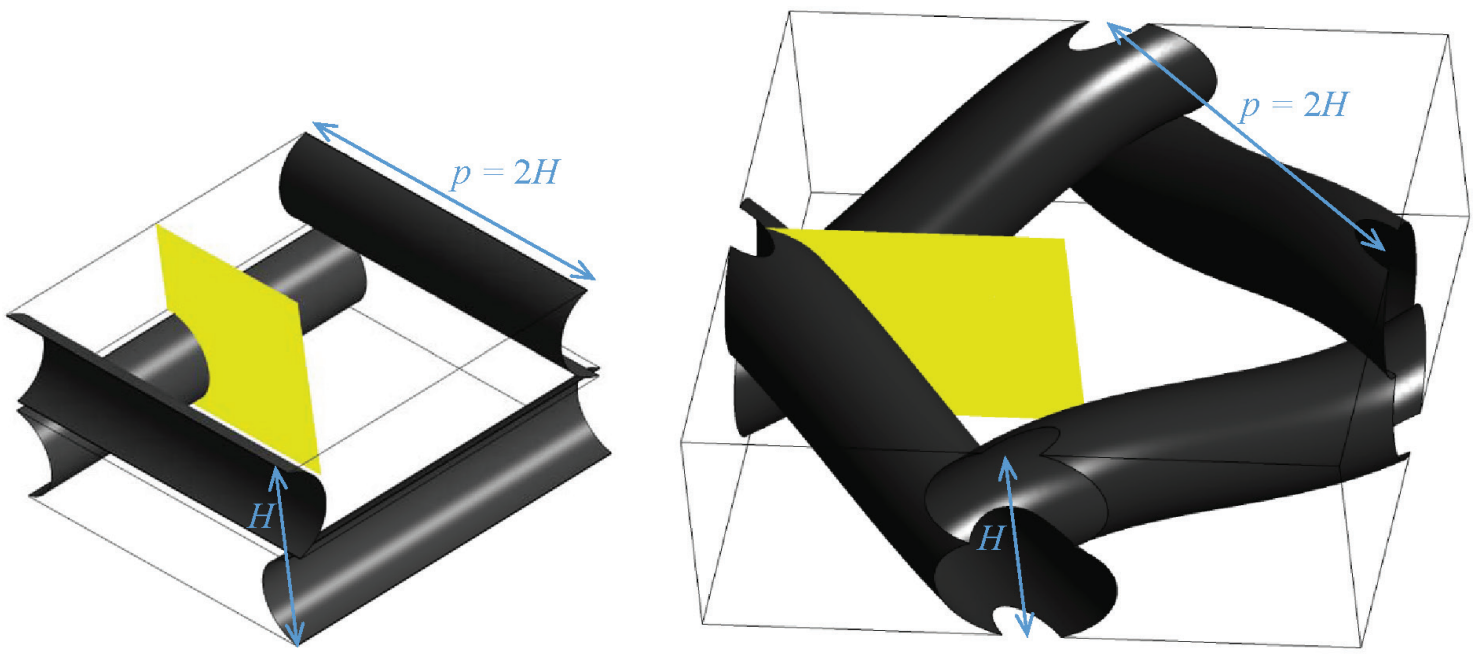

Fig. 6. Unit cells adopted as the computational domain for overlapped spacers (a) and woven spacers (b). The highlighted regions are those for which details of the computational finite volume grids are reported in Fig. 7.

$992.5 \mathrm{~kg} / \mathrm{m}^{3}, c_{p}=4186 \mathrm{~J} /(\mathrm{kgK}), k=0.5985 \mathrm{~W} /(\mathrm{mK}), m=6.625$ $\times 10^{-4} \mathrm{~Pa} \times \mathrm{s}$, yielding a Prandtl number of 4.63 . Finally, as in the parallel experiments, different orientations $\theta$ of the main flow with respect to the spacer filaments of the upper layer (flow attack angles) were considered $\left(0^{\circ}, 90^{\circ}\right.$ and $45^{\circ}$ for the overlapped spacer, $0^{\circ}$ and $45^{\circ}$ for the woven one).

For overlapped spacers, preliminary studies [36] showed that for the present pitch to height ratio of 2 the flow is steady up to friction velocity Reynolds numbers Re of $\sim 50$, corresponding to bulk Reynolds numbers of $\sim 300$ with a slight dependence on the flow attack angle. The same studies showed that, in this range, grid-independent results were obtained by using $\sim 4 \times 10^{5}$ finite volumes. Therefore, in this range simulations were conducted in steady-state mode, while the number of finite volumes was conservatively increased to $\sim 1 \times 10^{6}$. Purely hexahedral volumes were used; Fig. 7(a) reports a detail of the grid, corresponding to the highlighted region in Fig. 6(a).
In the case of unsteady flow ( $\operatorname{Re}>300)$, a limited number of direct numerical simulations were conducted using a much finer grid ( 5.5 million volumes) and protracting the simulations until statistically steady conditions were attained and well converged statistics could be computed. Details have been given in a previous paper [36]

For woven spacers, preliminary studies [38] showed that for the present pitch to height ratio of 2 the flow is steady up to friction velocity Reynolds numbers $\operatorname{Re}_{\tau}$ of $\sim 80$, corresponding to bulk Reynolds numbers of $\sim 240-270$ depending on the flow attack angle. The same studies showed that, in this range, grid-independent results were obtained by using $\sim 10^{6}$ finite volumes. Therefore, in this range simulations were conducted in steady-state mode, while the number of finite volumes was conservatively increased to $\sim 1.7 \times$ $10^{6}$. Unlike the corresponding overlapped-spacer geometry, the present one could not be discretized with purely hexahedral volumes; a hybrid grid was thus used, in which tet-

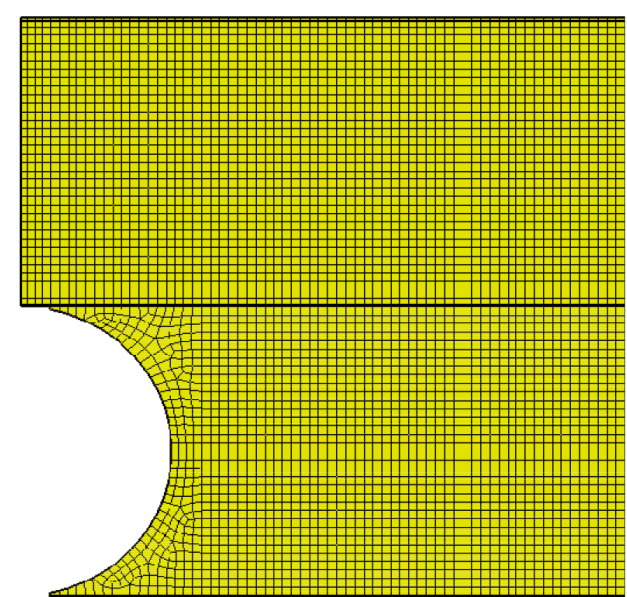

(a)

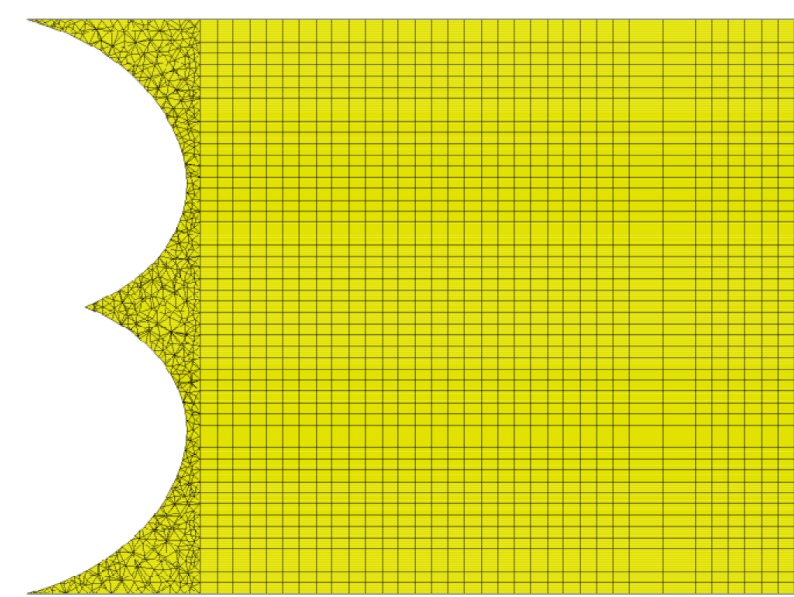

(b)

Fig. 7. Details of the computational grids (the regions shown are those highlighted in Fig. 6). (a) Overlapped spacers, steady-state conditions ( $N \approx 1$ million cells); (b) Woven spacers, all conditions ( $N \approx 1.7$ million cells). Finer grids $(N \approx 5.5$ million cells) were used for DNS of overlapped spacers. 
rahedral volumes were used in the regions surrounding the filaments ( $\sim 30 \%$ of the overall volume of the computational domain) and hexahedral volumes in the remaining regions. Fig. 7(b) reports a detail of the computational grid, corresponding to the highlighted region in Fig. 6(b).

For larger values of $\mathrm{Re}_{\tau}$ a time-dependent behaviour was observed, indicating the loss of stability of the base steady-state flow. Periodic flow was obtained for $\operatorname{Re}_{\tau} \approx 100$ 120 in the case $\theta=0^{\circ}$ (bulk Reynolds number $\sim 315-390$ ) and for $\operatorname{Re}_{\tau} \approx 90-110$ in the case $\theta=45^{\circ}$ (bulk Reynolds number $\sim 320-420)$. Simulations were also extended to incipient chaotic (turbulent) flow, obtained, in particular, for $\operatorname{Re}_{\tau}=140$ in the case $\theta=0^{\circ}$ (bulk Reynolds number 478) and for $\operatorname{Re}_{\tau}$ $=120$ in the case $\theta=45^{\circ}$ (bulk Reynolds number 465). For these cases, however, the number of grid cells was not incremented. A computational grid of "just" 1.7 million cells may appear inadequate to provide a full resolution of the turbulence structures; however, it should be considered that the present turbulence differs deeply from that typical of simple channels, occurs at much lower Reynolds numbers, and is characterized by larger spatial and temporal scales. As it will be shown below, DNS predictions for turbulent flow do not exhibit any change of trend with respect to lower-Re, steady-state or periodic, results and show a comparable agreement with experimental data.

\section{Comparative results}

Most of the results presented in this Section have been presented in references [36] in regard to overlapped spacers and [38] in regard to woven spacers. However, a unified comparison of the two basic configurations has not been published so far.

\subsection{Local Nusselt number distributions}

By way of example, Fig. 8 reports experimental (left) and computed (right) distributions of the local Nusselt number on the top wall for the overlapped spacer at different flow attack angles $\theta$ and comparable Reynolds numbers Re. The insets show the flow direction and the arrangement of the spacer filaments; note that these form two mutually orthogonal overlapped arrays, and that the filaments of the lower layer have line contacts with the bottom (adiabatic) wall while those of the upper layer have line contacts with the top (thermally active) wall. All the cases illustrated here correspond to turbulent flow; in order to reduce irregularities, experimental images were averaged over six time instants (separated by 2-3 s) and six distinct unit cells. Computational results were obtained by direct numerical simulation using the fine grid (5.5 million cells), and represent time averages taken over 20,000 time steps.

Plot (a) shows the experimental results obtained for $\theta$ $=0^{\circ}$ and a flow rate $Q=200 \mathrm{l} / \mathrm{h}$, yielding $\operatorname{Re} \approx 596 . \mathrm{Nu}$ is largest in the central region of the cell, with shallow maxima of $\sim 25$ just downstream of each spacer filament of the lower layer (minimum of the flow cross section), and decreases, though not monotonically, as one moves spanwise towards the contact lines with the spacer filaments of the upper layer, where heat transfer occurs only by conduction and very low minima of $\mathrm{Nu}$ are attained. The wall-av- eraged Nusselt number for this case was $\langle\mathrm{Nu}\rangle \approx 15$.3. Plot (b) reports time-averaged DNS results for the same $\theta=0^{\circ}$ and a similar Reynolds number $\operatorname{Re} \approx 607$, corresponding to an imposed friction velocity Reynolds number $\operatorname{Re}_{\tau}=80$. On the whole, the $\mathrm{Nu}$ distribution is similar to the experimental one, though of course much more regular and symmetric. For this case, the wall-averaged Nusselt number was $\langle\mathrm{Nu}\rangle$ $\approx 17.6, \sim 15 \%$ higher than the experimental value. Taking account of the small difference in Re (about $+2 \%$ ) and of the fact that $\langle\mathrm{Nu}\rangle$ increases with Re following roughly a 0.5 power law, the difference in $\langle\mathrm{Nu}\rangle$ is $\sim+14 \%$.

Plot (c) shows experimental results for $\theta=90^{\circ}$ and a flow rate $Q=225 \mathrm{l} / \mathrm{h}(\mathrm{Re} \approx 667)$. Nu maxima of $\sim 60$ are attained shortly upstream of each spacer filament touching the active wall, which in this case is a transverse obstacle for the main flow. Note that $\mathrm{Nu}$ values are not spanwise uniform as they would be in the absence of the longitudinal filaments, but attain two marked maxima, located symmetrically with respect to the midline and roughly midway between this and the spacer filaments of the lower layer. A large fraction of the wall is interested by low heat transfer rates $(\mathrm{Nu}<15)$, and thus contributes little to the overall thermal performance of the channel, which is not much different than for $\theta=0^{\circ}$. In fact, in this case one has $\langle\mathrm{Nu}\rangle \approx 18.4$, which is $\sim 14 \%$ higher than that obtained for $\theta=0^{\circ}(15.3)$ taking account of the different $\operatorname{Re}(667$ against 596). Plot (d) reports DNS results for the same friction velocity Reynolds number $\operatorname{Re}_{\tau}=80$ as in the previous case, yielding also the same Reynolds number $(\operatorname{Re} \approx 607)$ since the friction coefficient does not change for a $90^{\circ}$ rotation of the flow direction. Although the overall distribution of $\mathrm{Nu}$ is similar to the experimental one, there is a strong overprediction of $\mathrm{Nu}$ in the regions of maxima, which are in this case higher than 80 . However, since these regions are small, the wall-averaged Nusselt number is $\langle\mathrm{Nu}\rangle \approx 18.4$, nominally identical to the experimental result but actually corresponding to an overprediction of $5-6 \%$ once the difference in Re (607 against $667)$ is taken into account.

Finally, plot (e) reports experimental results for $\theta=45^{\circ}$ and $\operatorname{Re} \approx 690$. The Nu distribution shows maxima shortly upstream of the top spacer filament (which, in this case, acts as an oblique obstacle with respect to the main flow) and characteristic undulations of the iso-Nu lines. The wall average of $\mathrm{Nu}$ is $\langle\mathrm{Nu}\rangle \approx 29.8$, considerably higher (60-80\%) than in the previous arrangements with $\theta=0^{\circ}$ or $90^{\circ}$. The insets show the main flow direction and the filaments' arrangement. Finally, plot (f) shows DNS results for the same angle $\theta=45^{\circ}$ and $\operatorname{Re} \approx 530$, obtained again for $\operatorname{Re}_{\tau}=80$ (the lower Re is due to the higher value of the friction coefficient).

The insets show the main flow direction and the filaments' arrangement.

As in the previous case $\theta=90^{\circ}$, some disagreement with the experiments exists in the maximum values, which are higher than $\sim 80$ according to DNS but do not exceed $\sim 70$ in the experiments; however, the surface-averaged value of $\mathrm{Nu}$ is 26.2, very close to the experimental value (29.8) taking account of the lower Re (530 against 690).

Fig. 9 reports experimental (left) and computed (right) distributions of the local Nusselt number on the top wall for the woven spacer at flow attack angles $\theta=0^{\circ}$ or $45^{\circ}$ and comparable Reynolds numbers. As in the previous figure, the insets show the flow direction and the arrangement of 


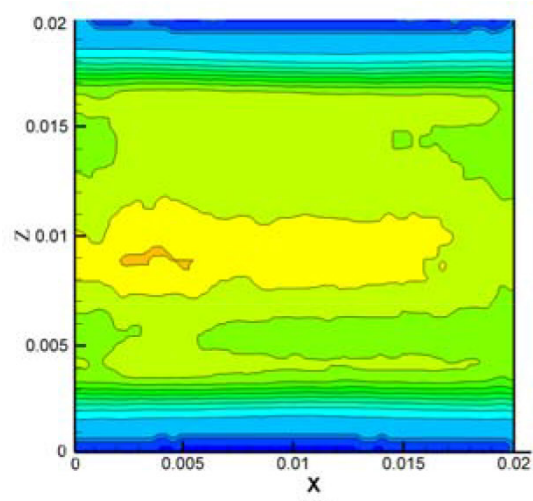

(a)

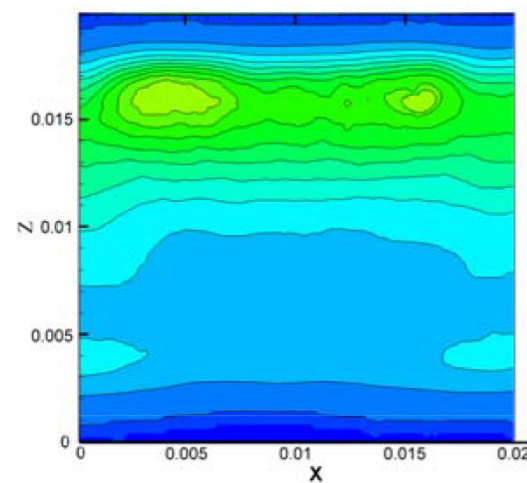

(c)

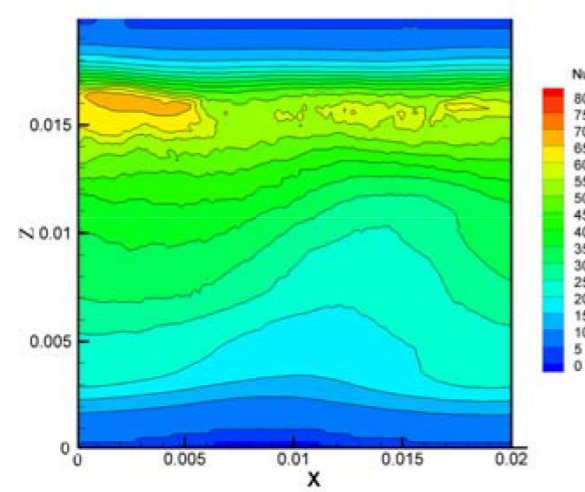

(e)

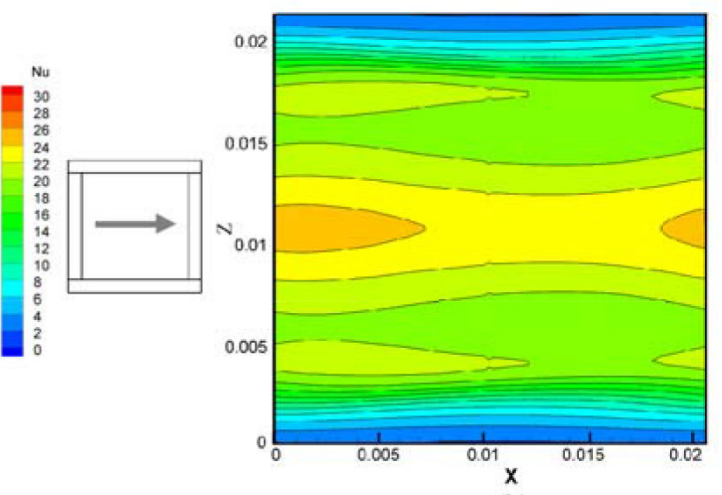

(b)

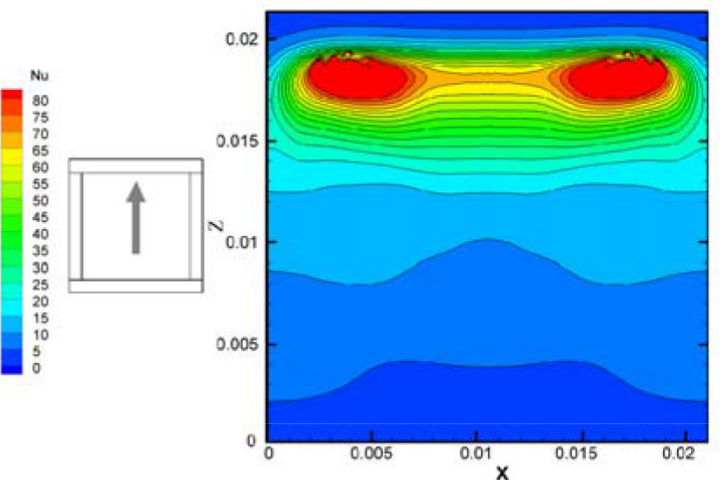

(d)

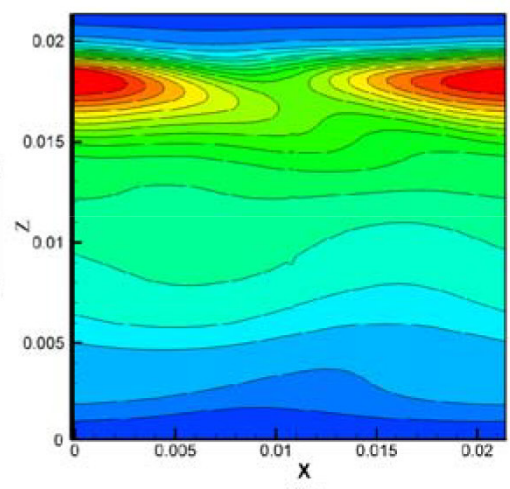

(f)

Fig. 8. Experimental (left) vs. computed (right) distributions of the local Nusselt number on the top wall for the overlapped spacer at different flow attack angles and comparable Re. (a) Experimental, $\operatorname{Re} \approx 596, \theta=0^{\circ}(\langle\mathrm{Nu}\rangle \approx 15.3) ;(\mathrm{b}) \mathrm{DNS}, \operatorname{Re} \approx 607, \theta=0^{\circ}(\langle\mathrm{Nu}\rangle \approx$ $17.6)$; (c) Experimental, $\operatorname{Re} \approx 667, \theta=90^{\circ}(\langle\mathrm{Nu}\rangle \approx 18.4)$; (d) DNS, $\operatorname{Re} \approx 607, \theta=90^{\circ}(\langle\mathrm{Nu}\rangle \approx 18.4)$; (e) Experimental, $\operatorname{Re} \approx 690, \theta=45^{\circ}(\langle\mathrm{Nu}\rangle$ $\approx 29.8$; (f) DNS, $\operatorname{Re} \approx 530, \theta=45^{\circ}(\langle\mathrm{Nu}\rangle \approx 26.2)$.

the spacer filaments; note that the line contacts characteristic of overlapped spacers are now replaced by point contacts, deformed into small areas both in the experiments (due to the filament elasticity) and in the simulations (in order to avoid grid singularities). Also for this configuration the cases illustrated correspond to turbulent flow.

The insets show the main flow direction and the filaments' arrangement.

Computational results, graphs (b) and (d), were obtained by direct numerical simulation using, as discussed above, a grid of $~ 1.7$ million cells. Unlike graphs (b), (d) and (f) in Fig. 8, which represent time averages, these are instantaneous fields extracted from the chaotic results at arbitrary time steps; this is reflected in the irregular shape of the contours. Graphic interpolation issues are present in these plots at the boundaries between regions gridded by tetrahedra and hexahedra.

In particular, graph (a) shows experimental results for $Q=150 \mathrm{l} / \mathrm{h}(\operatorname{Re} \approx 520)$ and $\theta=0^{\circ}$, while graph $(\mathrm{b})$ shows corresponding DNS results for $\operatorname{Re}_{\tau}=140(\operatorname{Re}=478)$. Computational $\mathrm{Nu}$ distributions and levels are close to their experimental counterparts; the main differences regard the values of $\mathrm{Nu}$ maxima, which are somewhat overestimated. However, wall averaged values of $\mathrm{Nu}$ are very similar (50 in the experiments, 52 in the simulations). 


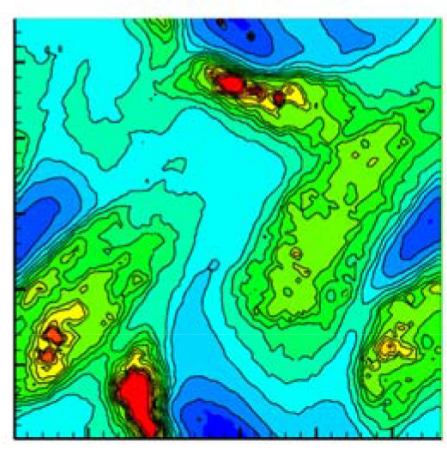

(a)

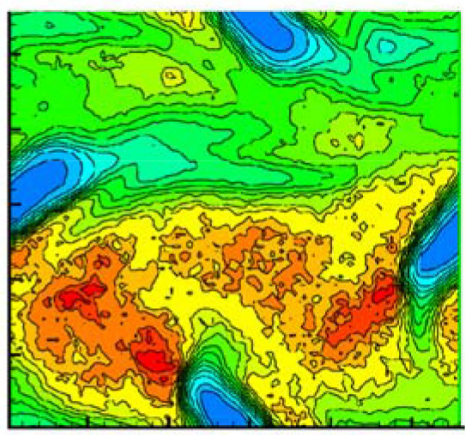

(c)
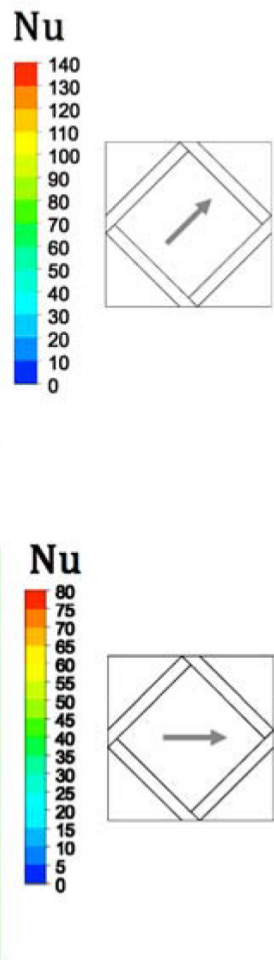

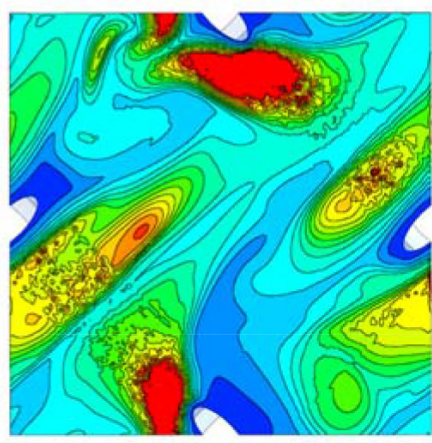

(b)

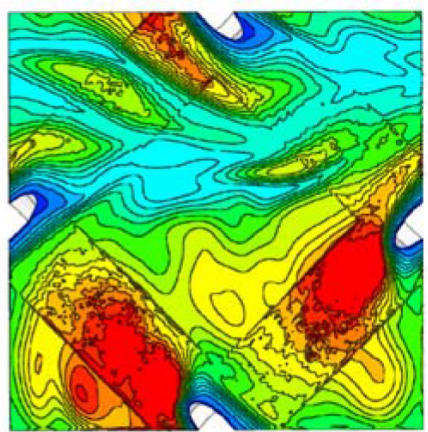

(d)

Fig. 9. Experimental (left) vs. computed (right) distributions of the local Nusselt number on the top wall for the woven spacer at different flow attack angles and comparable Re. (a) Experimental, $\operatorname{Re} \approx 520, \theta=0^{\circ}(\langle\mathrm{Nu}\rangle \approx 50) ;(\mathrm{b}) \mathrm{DNS}, \operatorname{Re} \approx 478, \theta=0^{\circ}(\langle\mathrm{Nu}\rangle \approx 52)$; (c) Experimental, $\operatorname{Re} \approx 540, \theta=45^{\circ}(\langle\mathrm{Nu}\rangle \approx 55)$; (d) DNS, $\operatorname{Re} \approx 465, \theta=45^{\circ}(\langle\mathrm{Nu}\rangle \approx 49)$.

Graph (c) shows experimental results for $\theta=45^{\circ}$ and $Q=156 \mathrm{l} / \mathrm{h}$, corresponding to $\operatorname{Re} \approx 540$, while graph $(\mathrm{d})$ reports corresponding DNS results for $\operatorname{Re}_{\tau}=120(\operatorname{Re}=465)$. Also for this orientation experimental and computational distributions and levels of $\mathrm{Nu}$ are in fair agreement (experimental $\langle\mathrm{Nu}\rangle=55$, predicted $\langle\mathrm{Nu}\rangle=49)$.

\subsection{Friction coefficient}

The friction coefficient $f$ is reported as a function of the Reynolds number for all cases investigated in Fig. 10.

Experimental results were obtained only for $\operatorname{Re}>\sim 100$; lower Re yield very small pressure drops, difficult to measure experimentally. In this range, for woven spacers and overlapped spacers at $\theta=45^{\circ}$ reliable results were obtained by our research group $[36,38]$ while, for overlapped spacers at $\theta=0^{\circ}-90^{\circ}$, results obtained by Li et al. [20] for a similar configuration (but with $P / H=2.2$ ), suitably converted to suit the present definitions of Re and $f$, are reported.

$\mathrm{Up}$ to $\mathrm{Re} \approx 20-30$ (i.e., under conditions of creeping flow), computational results follow almost exactly a $\operatorname{Re}^{-1}$ trend and show no difference between the various flow attack angles; woven spacers present friction coefficients more than two times higher than overlapped spacers. For larger $\mathrm{Re}$, they increasingly deviate from this trend and merge smoothly, especially for woven spacers at $\theta=0^{\circ}-90^{\circ}$, with the experimental results; some underprediction can be observed for woven spacers at $\theta=45^{\circ}$ and for overlapped

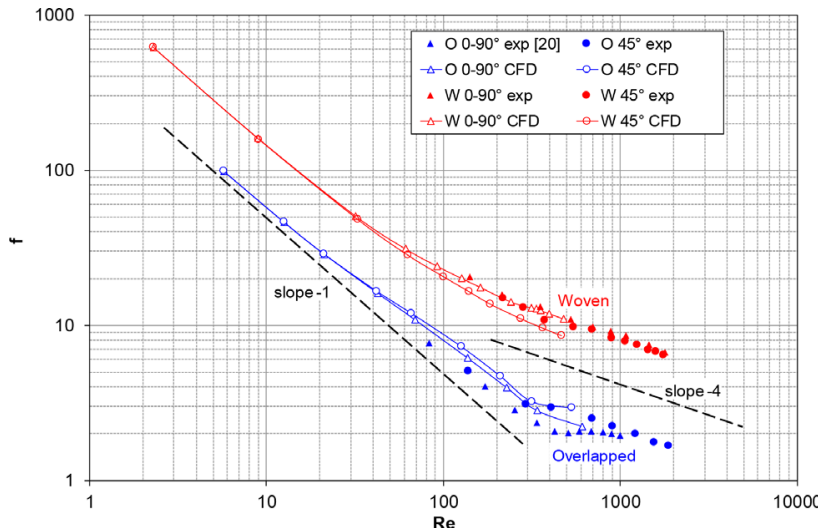

Fig. 10. Experimental and computational results for the Darcy friction coefficient $f$ as a function of the Reynolds number in overlapped and woven spacers at different flow attack angles.

spacers at both orientations. For Re larger than $\sim 1000$, when the flow is fully turbulent, results tend to follow a power law trend with an exponent of $\sim-0.4$; in this range, the influence of the flow attack angle becomes again small and the ratio between $f$ values for the woven and the overlapped configurations tends to $\sim 4$. In the transitional range $\operatorname{Re} \approx$ 300-1000 between steady and fully turbulent flow no jump occurs in the values of $f$ for any of the configurations investigated here, which confirms that, in complex geometries, 
transition occurs smoothly (in bifurcation theory language, supercritically). What is observed is rather an inflection point in the $f(\operatorname{Re})$ curves, which is particularly evident in the case of overlapped spacers at $0^{\circ}-90^{\circ}$, where a plateau in $f$ can be observed between $\operatorname{Re} \approx 500$ and 800 .

\subsection{Average Nusselt number}

Fig. 11 reports experimental and computational values of the top-wall averaged Nusselt number $\langle\mathrm{Nu}\rangle$ as a function of the Reynolds number for all the configurations examined.

For the overlapped spacer (blue symbols and lines), experimental results were obtained in the range $\operatorname{Re} \approx 140-$ 1850. The orientations $\theta=90^{\circ}$ (solid squares) and $\theta=0^{\circ}$ (solid triangles) present almost the same Nusselt numbers for all values of Re tested. On the other hand, the arrangement at $45^{\circ}$ (solid circles) presents significantly larger values of $\langle\mathrm{Nu}\rangle$. For all orientations, at Re $>\sim 1000$ the dependence of $\langle\mathrm{Nu}\rangle$ on $\mathrm{Re}$ can be approximated by a power law with an exponent close to 0.5. This exponent is similar to that $(\sim 0.43)$ reported by Tamburini et al. [33] for a similar but commercial, smaller-scale, Diamond spacer and also to that (0.5) appearing in the correlation presented by Da Costa et al. [17] for mass transfer and subsequently adopted by Phattaranawik et al. [26] for heat transfer on the basis of the heat-mass transfer analogy. At the lower end of the experimental range $(\operatorname{Re}<\sim 300)$ the increase of $\langle\mathrm{Nu}\rangle$ is weaker. The intermediate range $(\mathrm{Re}=300-1000)$ exhibits a steep increase of $\langle\mathrm{Nu}\rangle$, typical of transition to turbulence.

For the same overlapped spacer, computational results (hollow symbols) obtained under steady-state assumptions start from $\operatorname{Re} \approx 5-6$, extend up to $\operatorname{Re} \approx 300$ and exhibit a complex behaviour. At the lower end of the range investigated ( $\operatorname{Re}<20-30)$ the dependence of $\langle\mathrm{Nu}\rangle$ on Re is very weak, as expected from previous studies [39], which indicates that inertial effects and secondary flows play but a minor role. In this range, the values of $\langle\mathrm{Nu}\rangle$ for $\theta=0^{\circ}$ and $45^{\circ}$ are close to the exact (analytical) $\mathrm{Nu}$ for parallel flow in a plane (void, or spacerless) channel, which we calculated to be $\sim 5.15$ for one-side heat transfer under the present boundary conditions; for $\theta=$ $90^{\circ},\langle\mathrm{Nu}\rangle$ is even lower than the spacerless value, indicating that under these conditions the spacer behaves as an obstacle

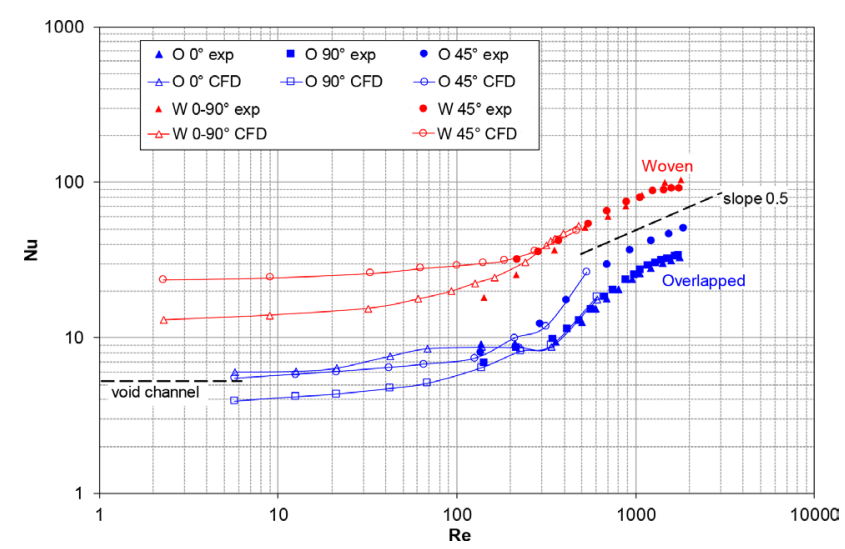

Fig. 11. Experimental and computational results for the top wall averaged Nusselt number $\langle\mathrm{Nu}\rangle$ as a function of the Reynolds numbers in overlapped and woven spacers at different flow attack angles. to heat transfer, rather than as a promoter. For $\operatorname{Re}>20-30$, different orientations present a different behaviour: for $\theta=0^{\circ}$ $\langle\mathrm{Nu}\rangle$ exhibits between $\operatorname{Re} \approx 60$ and $\operatorname{Re} \approx 300$ a plateau of $\sim 8.6$, which is missing for $\theta=90^{\circ}$ and $\theta=45^{\circ}$. As a consequence, the $\langle\mathrm{Nu}\rangle-\mathrm{Re}$ curve computed for $\theta=0$ (hollow triangles), which is the highest of the three for very low $\mathrm{Re}$, crosses those relative to the other two orientations and eventually becomes the lowest for $\operatorname{Re}>\sim 300$. This complex behavior is in good agreement with the experimental results, at least in the limited range of superposition $(\operatorname{Re} \approx 100-300)$. Finally, for $\operatorname{Re}>300$, the average Nusselt number for all three orientations (computed by direct numerical simulations) acquires a markedly increasing trend, in agreement with the experiments.

For the woven spacer (red symbols and lines), experimental results indicate that the orientation $\theta=0^{\circ}-90^{\circ}$ (equivalent in the woven arrangement, and indicated by solid triangles) and the orientation $\theta=45^{\circ}$ yield about the same values of $\langle\mathrm{Nu}\rangle$ for $\mathrm{Re}>\sim 400$, while for lower Re the orientation $\theta=45^{\circ}$ seems to be slightly better. For $\operatorname{Re}>1000$, the $\langle\mathrm{Nu}\rangle-\mathrm{Re}$ dependence follows roughly the same 0.5 power law as for the overlapped spacer; in the transitional range $\mathrm{Re}=300-1000$ the increase of $\langle\mathrm{Nu}\rangle$ is less steep than in the overlapped case.

Computational results for the woven spacer (hollow symbols) start from a very low bulk Reynolds number (Re $\approx 2$ ). Under these conditions (creeping flow), $\langle\mathrm{Nu}\rangle$ is almost independent of $\mathrm{Re}$, as in parallel flow, but exhibits values depending on the flow attack angle and much higher than the overlapped spacer $\langle\mathrm{Nu}\rangle$ and also than the exact (analytical) $\mathrm{Nu}$ for parallel flow in a void (spacerless) channel. At Reynolds numbers for which experimental data are available for comparison, CFD results generally compare favourably with these latter, with only some overprediction $(\sim 20 \%)$ for the case at $\operatorname{Re} \approx 150$ and $\theta=0^{\circ}$ (which is the only experimental result obtained for such low flow rates), and show a similar trend with $\operatorname{Re}$. For $\operatorname{Re}>500$, only experimental data exist and they exhibit an approximate 0.5-power law Reynolds number dependence. Note that a mean Nusselt number approximately increasing as $\operatorname{Re}^{0.5}$ has been reported for different spacers by the present $[33,36]$ and other authors $[17,26]$.

On the whole, the woven spacer yields average Nusselt numbers 4-5 times higher than the overlapped spacer at the lower end of the Reynolds number range investigated (creeping flow at $\operatorname{Re}<20-30$ ) and 2.5-3 times higher at the upper end (turbulent flow at $\operatorname{Re}=1000-2000$ ). Note that this behaviour is somewhat complementary to that of the friction coefficient, for which the woven/overlapped ratio is higher at high Re than at low Re.

\subsection{Performance comparison for a given power consumption}

Since woven spacers simultaneously yield higher pressure drops and higher heat transfer coefficients than overlapped spacers, the choice between the two configurations depends crucially on the relative importance attributed to savings in membrane surface area and in pumping energy for any specific application. In the absence of information regarding the unitary cost of membrane surface and electric power, general conclusions regarding the relative merits of either configuration are impossible. However, a reasonably general comparison can be obtained by plotting the aver- 


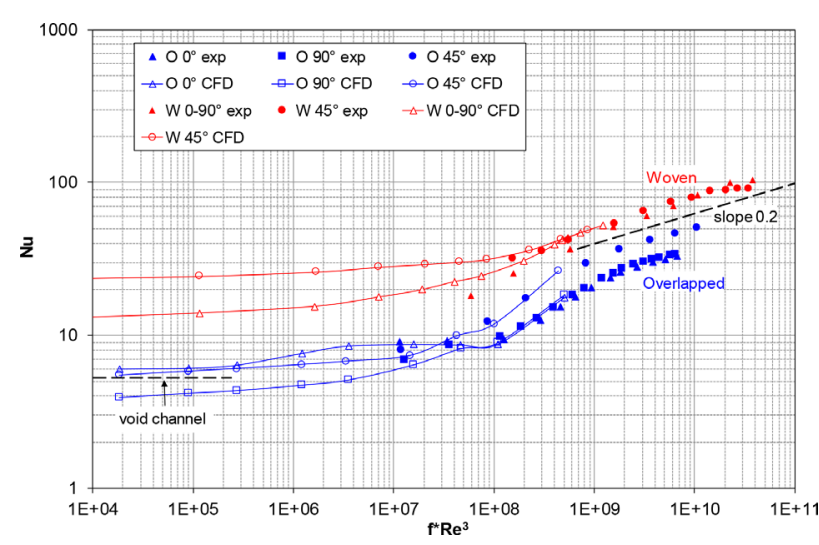

Fig. 12. Experimental and computational results for the top wall averaged Nusselt number $\langle\mathrm{Nu}\rangle$ as a function of the parameter $f$ $\operatorname{Re}^{3}$ (proportional to the specific pumping energy consumption per unit volume of feed) in overlapped and woven spacers at different flow attack angles.

age Nusselt number as a function of the product $f \operatorname{Re}^{3}$ (also called power number and denoted by Pn), a dimensionless parameter proportional to the specific pumping energy consumption per unit volume of feed.

Fig. 12 reports the average Nusselt number $\langle\mathrm{Nu}\rangle$ as a function of $f \operatorname{Re}^{3}$ for all configurations investigated. In this plot, for consistency reasons, experimental and computational $\langle\mathrm{Nu}\rangle$ data are associated with experimental and computational values of $f \operatorname{Re}^{3}$, respectively. It can be observed that, despite the higher pressure losses, woven spacers provide higher values of $\langle\mathrm{Nu}\rangle$ for any given value of $f \operatorname{Re}^{3}$ in the whole Reynolds number range, the advantage being maximum (about threefold) at low Reynolds numbers and minimum (about twofold) at the highest Re. Thus, in the absence of more detailed information on the relative costs of membrane surface and electric energy, woven spacers should be regarded as a more convenient alternative than overlapped ones.

\section{Conclusions}

Heat transfer and pressure drop characteristics of overlapped and woven spacers were compared on the basis of experiments with Thermochromic Liquid Crystals and parallel numerical simulations. The study was conducted for a pitch to height ratio of 2 , flow attack angles of $0^{\circ}, 45^{\circ}$ and $90^{\circ}$, and Reynolds numbers ranging from creeping to turbulent flow. Numerical simulations were based on a finite volume method and were conducted in steady-state mode for Reynolds numbers up to 300 and in unsteady mode (i.e., as direct numerical simulations) for larger Re. Only one of the walls was assumed to be thermally active, the opposite wall being adiabatic. On the active wall, mixed (Cauchy) thermal boundary conditions were assumed, mimicking the experimental setup.

A satisfactory agreement was obtained between experiments and simulations in regard to both the average Nusselt number $\langle\mathrm{Nu}\rangle$ and the Darcy friction coefficient $f$. The results showed that woven spacers provide higher Nusselt numbers but also higher friction coefficients. When the two parameters were combined by reporting the Nusselt number as a function of the power number $f \operatorname{Re}^{3}$, woven spacers were found to yield higher values of $\langle\mathrm{Nu}\rangle$ for any specific pumping power consumption. However, ultimately the choice between the two configurations will depend on the relative importance attributed to savings in membrane surface area rather than in pumping energy for any specific application.

\section{Acknowledgements}

This work was funded within the EU FP7 programme by project MEDIRAS (contract number 218938).

\section{Symbols}

$c_{p} \quad$ - Specific heat at constant pressure $\left[\mathrm{J} \mathrm{kg}^{-1} \mathrm{~K}^{-1}\right]$

$D_{h}$ - Hydraulic diameter of the void channel, $2 \mathrm{H}[\mathrm{m}]$

$f^{h}$ - Darcy friction coefficient [-]

$H$ - Channel height [m]

$h$ - Heat transfer coefficient $\left[\mathrm{W} \mathrm{m}^{-2} \mathrm{~K}^{-1}\right]$

$k$ - Thermal conductivity $\left[\mathrm{W} \mathrm{m} \mathrm{m}^{-1} \mathrm{~K}^{-1}\right]$

L - Thickness [m]

$\mathrm{Nu}$ - Local Nusselt number, $h 2 H / \kappa[-]$

$P \quad$ - Pitch (inter-filament distance) $[\mathrm{m}]$

Pn - Power number, $f \operatorname{Re}^{3}[-]$

$p_{\xi} \quad$ - Pressure gradient along the main flow direction $\left[\mathrm{Pa} \mathrm{m}^{-1}\right]$

$Q \quad$ - Volume flow rate $\left[\mathrm{m}^{3} \mathrm{~s}^{-1}\right.$ or $\left.1 \mathrm{~h}^{-1}\right]$

$q_{w}^{\prime \prime}$ - Wall heat flux $\left[\mathrm{W} \mathrm{m}^{-2}\right]$

${ }^{w}$ - Thermal resistance $\left[\mathrm{m}^{2} \mathrm{~K} \mathrm{~W}^{-1}\right]$

Re - Bulk Reynolds number, $U 2 H / v[-]$

$\operatorname{Re}_{\tau}-$ Friction velocity Reynolds number, $u_{\mathrm{t}}(H / 2) / v[-]$

$T^{\tau}$ - Temperature $[\mathrm{K}]$

TPC - Temperature polarization coefficient, see Eq. (1) [-]

$\mathrm{U}$ - Mean void-channel streamwise velocity, $Q /(W H)$ $\left[\mathrm{m} \mathrm{s}^{-1}\right]$

$u_{\tau} \quad$ - Friction velocity $\left[\mathrm{m} \mathrm{s}^{-1}\right]$

$W^{\tau}$ - Spanwise width of the experimental test section $[\mathrm{m}]$

\section{Greek}

$\mu$ - Dynamic viscosity [Pa s]

$v \quad$ - Cinematic viscosity $\left[\mathrm{m}^{2} \mathrm{~s}^{-1}\right]$

$\rho \quad-$ Density $\left[\mathrm{kg} \mathrm{m}^{-3}\right]$

$\theta \quad$ - Flow attack angle $\left[^{\circ}\right]$

$\tau \quad$ - Wall shear stress [Pa]

$\xi \quad$ - Distance along the main flow direction [m]

\section{Subscripts}

b - Bulk

c - Cold channel

$f$ - Feed

$h$ - Hot channel

$m$ - Membrane surface

$p$ - Permeate

TLC - Thermochromic Liquid Crystal sheet

$w$ - Wall

$\xi \quad$ - Main flow direction

\section{Averages}

$\langle\Phi\rangle$ - Area average of the generic quantity $\Phi$ on a wall 


\section{References}

[1] A. AlHathal Al-Anezi, A.O. Sharif, M.I. Sanduk, A.R. Khan Potential of membrane distillation - A comprehensive review, Int. J. Water, 7 (2013) 317-346.

[2] A. Cipollina, M.G. Di Sparti, A. Tamburini, G. Micale, Development of a membrane distillation module for solar energy seawater desalination, Chem. Eng. Res. Design, 90 (2012) 2101-2121.

[3] Y.-D. Kim, K. Thu, N. Ghaffour, K. Choon Ng, Performance investigation of a solar-assisted direct contact membrane distillation system, J. Membr. Sci., 427 (2013) 345-364.

[4] M.R. Qtaishat, F. Banat, Desalination by solar powered membrane distillation systems, Desalination, 308 (2013) 186197.

[5] M. Shatat, S.B. Riffat, Water desalination technologies utilizing conventional and renewable energy sources, Int. J. LowCarbon Technol., 9 (2014), 1-19.

[6] N. Palanisami, K. He, I.S. Moon, Utilization of solar energy for direct contact membrane distillation process: An experimental study for desalination of real seawater, Korean J. Chem. Eng., 31 (2014) 155-161.

[7] J. Koschikowski, M. Wieghaus, M. Rommel, Solar thermaldriven desalination plants based on membrane distillation, Desalination, 156 (2003) 295-304.

[8] J. Koschikowski, M. Wieghaus, M. Rommel, V.S. Ortin, B.P. Suarez, J.R. Betancort Rodríguez, Experimental investigations on solar driven stand-alone membrane distillation systems for remote areas, Desalination, 248 (2009) 125-131.

[9] R. Porrazzo, A. Cipollina, M. Galluzzo, G. Micale, A neural network-based optimizing control system for a seawaterdesalination solar-powered membrane distillation unit, Comp. Chem. Eng., 54 (2013) 79-96.

[10] R. Schwantes, A. Cipollina, F Gross, J. Koschikowski, D. Pfeifle, M. Rolletschek, V. Subiela, Membrane distillation: solar and waste heat driven demonstration plants for desalination, Desalination, 323 (2013) 93-106.

[11] M.S. El-Bourawi, Z. Ding, R. Ma, M. Khayet, A framework for better understanding membrane distillation separation process, J. Membr. Sci., 285 (2006) 4-29.

[12] Y.M.c. Manawi, M.a. Khraisheh, A.K.c. Fard, F.a. Benyahia, S Adham, Effect of operational parameters on distillate flux in direct contact membrane distillation (DCMD): Comparison between experimental and model predicted performance, Desalination, 336 (2014) 110-120.

[13] A. Ali, F. Macedonio, E. Drioli, S. Aljlild, O.A. Alharbi, Experimental and theoretical evaluation of temperature polarization phenomenon in direct contact membrane distillation, Chem. Eng. Res. Des., 91 (2013) 1966-1977.

[14] L. Martínez-Diez, J.M. Rodríguez-Maroto, Temperature and concentration polarization in membrane distillation of aqueous salt solutions, J. Membr. Sci., 156 (1999) 265-273.

[15] F. Laganà, G. Barbieri, E. Drioli, Direct contact membrane distillation: modelling and concentration experiments, J. Membr. Sci., 166 (2000) 1-11.

[16] J. Zuo, S. Bonyadi, T.S. Chung, Exploring the potential of commercial polyethylene membranes for desalination by membrane distillation, J. Membr. Sci., 497 (2016) 239-247.

[17] A.R. Da Costa, A.G. Fane, D.E. Wiley, Spacer characterization and pressure drop modelling in spacer filled channel for ultrafiltration, J. Membr. Sci., 87 (1994) 79-98

[18] G. Schock, A. Miquel, Mass transfer and pressure loss in spiral wound modules, Desalination, 64 (1987) 339-352.

[19] M. Shakaib, S.M.F. Hasani, I. Ahmed, R.M. Yunus, A CFD study on the effect of spacer orientation on temperature polarization in membrane distillation modules, Desalination, 284 (2012) 332-340.
[20] F. Li, W. Meindersma, A.B. de Haan, T. Reith, Experimental validation of CFD mass transfer simulations in flat channels with non-woven net spacers, J. Membr. Sci. 232 (2004) 19-30.

[21] A. Velázquez, J.I. Mengual, Temperature polarization coefficients in membrane distillation, Ind. Eng. Chem. Res., 34 (1995) 585-590.

[22] L. Martínez-Diez, M.I. Vazquez-González, F.J. Florido-Díaz, Study of membrane distillation using channel spacers, J. Membr. Sci., 144 (1998) 45-56.

[23] Y. Yun, J. Wang, R. Ma, A.G. Fane, Effects of channel spacers on direct contact membrane distillation, Desal. Water Treat., 34 (2011) 63-69.

[24] J. Phattaranawik, R. Jiraratananon, A.G. Fane, C. Halim, Mass flux enhancement using spacer filled channels in direct contact membrane distillation, J. Membr. Sci., 187 (2001) 193-201.

[25] J. Phattaranawik, R. Jiraratananon, A.G. Fane, Heat transport and membrane distillation coefficients in direct contact membrane distillation, J. Membr. Sci., 212 (2003) 177-193.

[26] J. Phattaranawik, R. Jiraratananon, A.G. Fane, Effects of net-type spacers on heat and mass transfer in direct contact membrane distillation and comparison with ultrafiltration studies, J. Membr. Sci., 217 (2003) 193-206.

[27] M.N.Chernyshov, G.W. Meindersma, A.B. de Haan, Comparison of spacers for temperature polarization reduction in air gap membrane distillation, Desalination, 183 (2005) 363-374.

[28] L. Martínez-Diez, J.M. Rodríguez-Maroto, Characterization of membrane distillation modules and analysis of mass flux enhancement by channel spacers, J. Membr. Sci., 274 (2006) 123-137.

[29] S. Al-Sharif, M. Albeirutty, A. Cipollina, G. Micale, Modelling flow and heat transfer in spacer-filled membrane distillation channels using open source CFD code, Desalination, 311 (2013) 103-112.

[30] A. Cipollina, A. Di Miceli, J. Koschikowski, G. Micale, L. Rizzuti, CFD simulation of a membrane distillation module channel, Desal. Water Treat., 6 (2009) 177-183.

[31] A. Cipollina, G. Micale, L. Rizzuti, Membrane Distillation heat transfer enhancement by CFD analysis of internal module geometry, Desal. Water Treat., 25 (2011) 195-209.

[32] M. Shakaib, S.M.F. Hasani, M. Ehtesham-ul Haque, I. Ahmed, R.M. Yunus, A CFD study of heat transfer through spacer channels of membrane distillation modules, Desal. Water Treat., 51 (2013) 3662-3674.

[33] A. Tamburini, P. Pitò, A. Cipollina, G. Micale, M. Ciofalo, A thermochromic liquid crystals image analysis technique to investigate temperature polarization in spacer-filled channels for membrane distillation, J. Membr. Sci., 447 (2013) 260-273.

[34] A. Tamburini, G. Micale, M. Ciofalo, A. Cipollina, Experimental analysis via thermochromic liquid crystals of the temperature local distribution in membrane distillation modules, Chem Eng. Trans., 32 (2013) 2041-2046.

[35] N. Abdullah, A.R. Abu Talib, A.A. Jaafar, M.A.M. Salleh, The basics and issues of thermochromic liquid crystal calibrations, Experim. Thermal Fluid Sci., 34 (2010) 1089-1121.

[36] A. Tamburini, M. Renda, A. Cipollina, G. Micale, M. Ciofalo, Investigation of heat transfer in spacer-filled channels by experiments and direct numerical simulations, Int. J. Heat Mass Trans., 93 (2016) 1190-1205.

[37] Ansys Inc., Ansys-CFX Reference Guide, Release 14.5 (2012).

[38] F. Ponzio, A. Tamburini, A. Cipollina, G. Micale, M. Ciofalo, Experimental and computational investigation of heat transfer in channels filled by woven spacers, Int. J. Heat Mass Trans. 104 (2017) 163-177.

[39] L. Gurreri, A. Tamburini, A. Cipollina, G. Micale, M. Ciofalo, Flow and mass transfer in spacer-filled channels for reverse electrodialysis: a CFD parametrical study, J. Membr. Sci., 497 (2016) 300-317. 